\title{
Treatment of recurrent myopic macular hole using membrane of plasma rich in growth factors
}

This article was published in the following Dove Press journal:

International Medical Case Reports Journal

\section{Ronald M Sánchez-Ávila \\ Álvaro Fernández- \\ Vega González \\ Álvaro Fernández-Vega Sanz \\ Jesús Merayo-Lloves}

University Institute Fernández-Vega, Ophthalmological Research Foundation, Oviedo, Spain
Correspondence: Ronald M Sánchez-Ávila University Institute Fernández-Vega, Ophthalmological Research Foundation, Avda Dres Fernández-Vega num 34,

Oviedo E-33012, Spain

Tel +3498524014l

Fax +34 985233288

Email ronald.sanchezavila@gmail.com
Purpose: To describe the case of a patient with a recurrent large myopic macular hole $(\mathrm{MH})$, who was successfully treated with a plasma rich in growth factors (PRGF) membrane. Case Report: A 71-year-old patient presented a myopic $\mathrm{MH}$ in his right eye. One month later, pars plana vitrectomy with internal limiting membrane (ILM) peeling was performed, achieving closure of the MH. Three months later a recurrence was observed $(700 \mu \mathrm{m})$ without visual acuity (VA) improvement. A new surgery was carried out placing an autologous PRGF-membrane into the $\mathrm{MH}$, and performing a fluid-gas exchange at the end of the surgery. After two months of follow-up, the $\mathrm{MH}$ was completely closed, obtaining the anatomic recovery of the foveal depression, and improving the VA to 0.1 . No adverse reactions were associated with the use of PRGF and there were no new recurrences of the $\mathrm{MH}$ in a follow-up period of six months.

Conclusion: The use of PRGF-membrane can be used as adjunctive therapy in the surgical repair of recurrent large myopic MHs, thus improving anatomic and visual outcomes.

Keywords: myopic macular hole, plasma rich in growth factors, PRGF, retinal surgery, macular hole

\section{Introduction}

Macular hole $(\mathrm{MH})$ is a common clinical finding, in patients with high myopia. The pathophysiology of myopic MHs is not fully understood; however, it is postulated that the myopic maculopathy traction may be the cause of this disease, ${ }^{1}$ the participation of epiretinal membranes, the rigidity of the internal limiting membrane (ILM) and the rigid retinal vessels are situations that promote the development of $\mathrm{MH}$ in myopic patients. ${ }^{2}$ Pars plana vitrectomy (PPV) is the most commonly used treatment for $\mathrm{MH}$ being associated with ILM peeling, acting on the inner retina to release the tensile forces. ${ }^{3}$ In the treatment of recurrent myopic $\mathrm{MH}$, new surgical techniques are used (inverted ILM flap technique and the autologous transplantation of ILM), which seems to improve the final postopera-

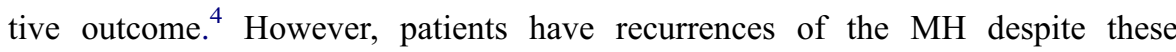
surgical techniques.

Plasma rich in growth factors (PRGF) is a novel autologous ophthalmic therapy that contains a large number of biologically active agents, including EGF (epidermal growth factor), PDGF (platelet-derived growth factor), insulin-like growth factor 1 , fibronectin and vitamin $\mathrm{A},{ }^{5}$ that promote tissue regeneration, healing modulation and anti-inflammatory properties, among others. ${ }^{5}$ PRGF-Endoret technology can be used to obtain different formulations, like eye drops and membranes, for the treatment of several ophthalmic diseases. ${ }^{6}$ The PRGF membrane has similar 
mechanical and biological properties to the amniotic membrane ${ }^{7}$ and it has been used in ocular and corneal surface surgery with good clinical results. ${ }^{8}$

The efficacy and safety of PRGF therapy have been widely demonstrated, particularly in dry eye syndrome, persistent epithelial defects, and corneal ulcers. ${ }^{9}$ Other authors have reported the efficacy of platelet derived (liquid use as supernatant) ${ }^{1}$ for the treatment of myopic MHs; however, to our knowledge this is the first report that uses the PRGF-membrane technology for the treatment of myopic $\mathrm{MH}$ closure.

\section{Case report}

A 71-year-old male patient with history of cataract surgery (previous refraction: -12.5 sphere, -1.00 to $0^{\circ}$ cylinder), with diffractive lens implant and high myopia (axial length of $30.19 \mathrm{~mm}$ ) in the right eye, developed a $\mathrm{MH} 15$ days after the cataract surgery, associated with metamorphopsia and decreased visual acuity (VA, count fingers). One month later, PPV with ILM peeling was performed achieving the closure of the $\mathrm{MH}$. Three months later, the visual symptoms returned (metamorphopsia), the VA had not improved (count fingers), and a $\mathrm{MH}$ recurrence was observed $(700 \mu \mathrm{m})$, associated with subretinal fluid, intraretinal hemorrhages, and pigment dispersion (Figure 1).

A new surgery was carried out in order to achieve the $\mathrm{MH}$ closure. The PRGF-membrane was prepared according to Endoret ${ }^{\circledR}$ technology (BTI Biotechnology Institute, S.L., Miñano, Álava, Spain). ${ }^{7}$ The surgical procedure consisted in enlarging the peeling of the ILM, close to the optic nerve and the vascular arcade and tap the edges of the $\mathrm{MH}$ (to increase the probability of closing the $\mathrm{MH}$, technique proposed by the retina surgeon: AFVS). Then, the PRGF membrane was introduced through the trocar and placed inside the $\mathrm{MH}$ (the thickness of the membrane was $100 \mu \mathrm{m}$ ), without pressure and without forcing the adhesion (PRGF fibrin membrane have adhesion properties when it comes into contact with the cell surface) trying to avoid the potential damage to the retinal pigment epithelium; subsequently three drops of liquid PRGF were placed in the $\mathrm{MH}$, and a fluid-gas (C3F8) exchange was performed at the end of the surgery (Figure 2).

To obtain the PRGF-membrane, $81 \mathrm{~mL}$ of the patient's blood was extracted in $9 \mathrm{~mL}$ tubes with $3.8 \%$ sodium citrate as an anticoagulant. The blood was centrifuged at $580 \mathrm{~g}$ at room temperature for 8 mins (Endoret system ${ }^{\circledR}$ ). The fraction 2 (F2) was defined as $2 \mathrm{~mL}$ of plasma just above the buffy coat and fraction 1 (F1) was defined as the remaining plasma above F2. To make each PRGF membrane, $5 \mathrm{~mL}$ of F2 was activated with $10 \%$ calcium chloride and then incubated in $35 \mathrm{~mm}$ diameter wells at $37^{\circ} \mathrm{C}$ for 30 mins. Once the fibrin clots were formed, they were placed in a shaping vessel and pressed for $30 \mathrm{~s}$, yielding $100 \mu \mathrm{m}$ thick PRGF-membrane. The F1 (liquid) was placed after the PRGF membrane was introduced into the $\mathrm{MH}$. This preparation does not contain cells.

After two months of follow-up, the $\mathrm{MH}$ was completely closed. Anatomic recovery of the foveal depression (assessed by SD-OCT) was observed and the mild intraretinal hemorrhages and subretinal fluid disappear, improving the VA to 0.1. An alteration was observed in the outer layers of the retina in the foveal area, but no adverse reactions were associated with the PRGF. We did not have any new recurrences of the $\mathrm{MH}$ in a follow-up period of six months, and VA remained stable during this period (Figure 3).


Figure I Retinography and optical coherence tomography (OCT) of recurrent myopic macular hole. (A) Retinography of the right eye showing the macular hole. (B) OCT of recurrent myopic macular hole in the right eye. 

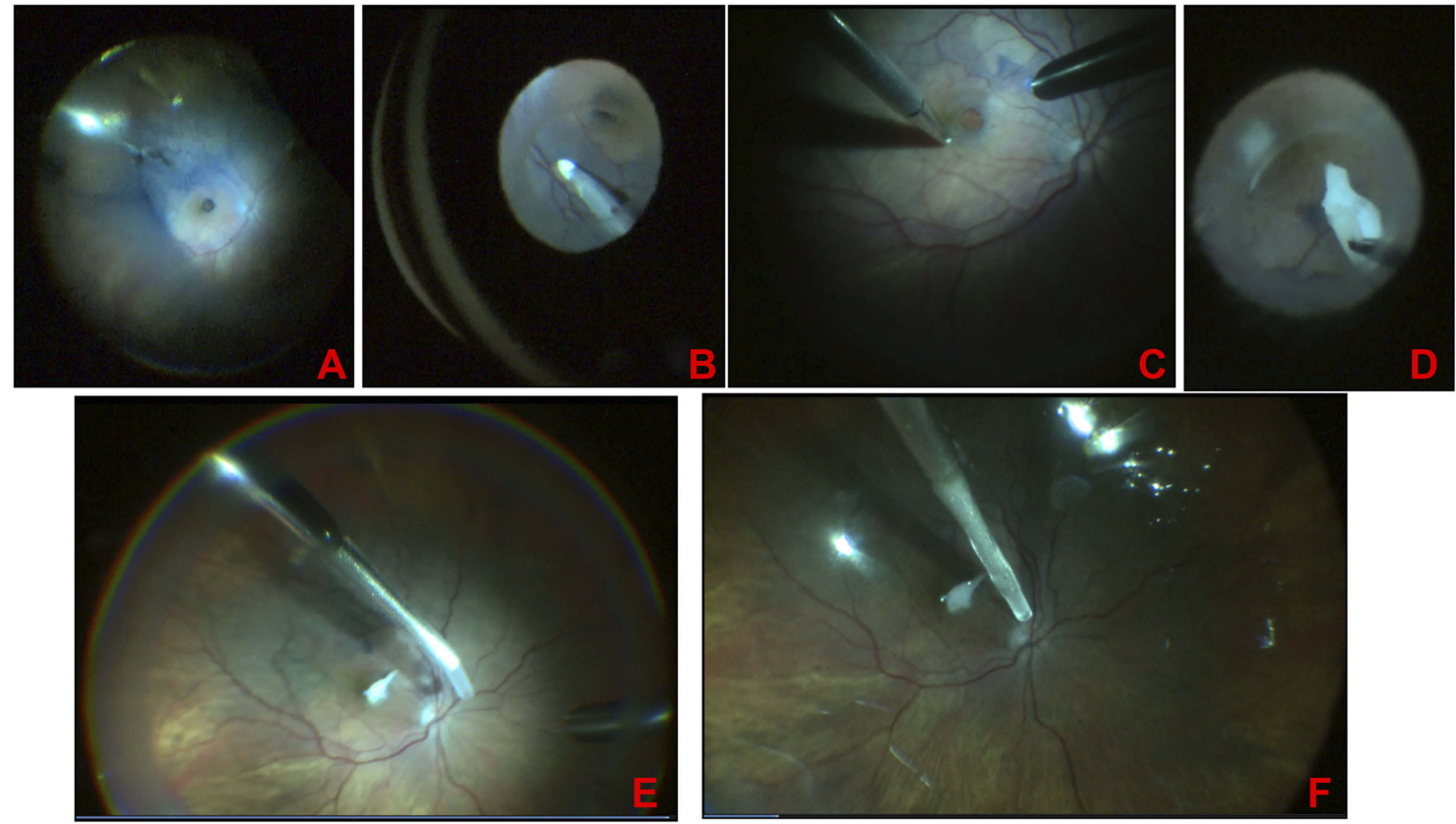

Figure 2 Surgery in the recurrent myopic macular hole (PPV + extended Internal Limiting Membran (ILM) peeling + PRGF-membrane). (A) Macular hole staining. (B) Extended ILM peeling. (C) Tap the edges of the macular hole. (D) PRGF-membrane introduction into vitreous cavity. (E) PRGF-membrane position into the macular hole. (F) fluid-air-gas exchange.

Abbreviations: PRGF, plasma rich in growth factors; PPV, pars plana vitrectomy.
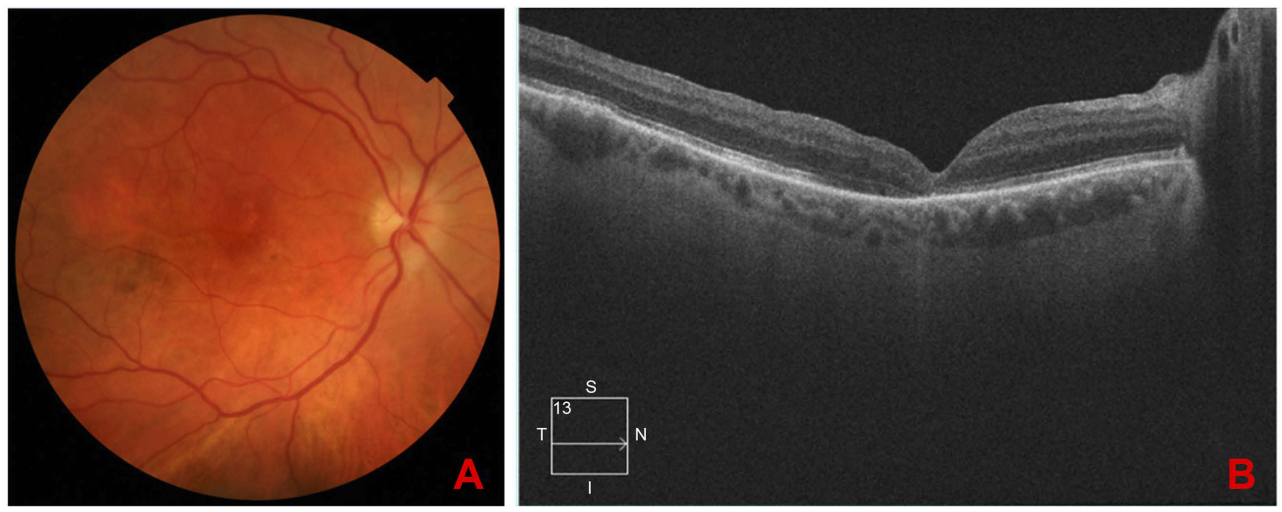

Figure 3 Post-surgical follow-up. (A) Three months after treatment with PPV + PRGF membrane. (B) Retinography of the right eye: Image of closed macular hole, macular pigmentary changes.

Abbreviations: PPV, pars plana vitrectomy; PRGF, plasma rich in growthfactor.

\section{Discussion}

The $\mathrm{MH}$ is a common finding in patients with high myopia, it is thought that its formation can be caused by the combination of tractional forces, at the macular level. ${ }^{1}$ The usual treatment for this pathology is PPV associated with procedures on ILM to decrease the tractional forces in the macula, ${ }^{1}$ such as the inverted ILM flap, the ILM peeling alone or with autologous PRPs (platelet-rich plasma) injection over the hole, autologous transplantation of ILM membrane, lens capsular flap, or neurosensory retina. ${ }^{10}$ In the inverted ILM flap technique, as well as in the autologous transplantation, the ILM acts as a scaffold for reactive gliosis and proliferation of glial cells, and therefore the anatomic closure of the $\mathrm{MH}$ is more easily produced. ${ }^{11}$ Likewise, the ILM could act as a barrier that prevents the penetration of fluids from the vitreous cavity to the macular orifice, preserving the function of the retinal pigment epithelium. However, the closure of the myopic MH is unpredictable, and a second surgery may be required. ${ }^{12}$ 
PRPs are platelet products suspended in plasma, which contain growth factors [such as PDGF, transforming growth factor- $\beta$, basic fibroblast growth factor (b-FGF), EGF, among others] and other molecules, which are involved in tissue regeneration and inflammatory modulation. ${ }^{6}$ There are different preparation protocols for PRPs; however, their clinical effectiveness is given by the platelet concentration, presence or absence of the leukocytes, the activation process, the production method (closed, open), and the storage process and dispensing. ${ }^{13}$ PRGF is a special type of PRP that uses Endoret ${ }^{\circledR}$ technology for its elaboration, with closed method, moderate concentration of platelets, absence of leukocytes, platelet activation, and storage up to three months, preserving the concentration of growth factors. ${ }^{6}$

The eye drops PRGF has shown in different in vitro, in vivo, and clinical studies to have a higher concentration of growth factors, proliferation capacity, migration and tissue regeneration in the ocular surface and cornea. ${ }^{14}$ The PRGF fibrin membrane has mechanical properties and resistance that allow its use in surgical procedures, it has been shown that inside of it contains a wide range of growth factors, which are released progressively over time. ${ }^{15,16}$ It has also been shown that the PRGF membrane serves as scaffolding for cell expansion in advanced therapy studies. ${ }^{7}$

In our case report, we describe a patient with myopic $\mathrm{MH}$, who had not responded to PPV surgery with ILM peeling; a second surgery was carried out with an extension of the ILM peeling margins, and with a PRGF membrane placement into the $\mathrm{MH}$. The anatomic closure and vision recovery were observed. There are some publications using liquid PRPs in myopic $\mathrm{MH},{ }^{1}$ however our case report would be the first one which uses an autologous fibrin membrane (PRGF) as adjuvant therapy to retinal surgery. The mechanism involved in the closure of the $\mathrm{MH}$ could be due to the trophic action of the growth factors (PDGF, b-FGF, EGF), which enhance the activation of Müller cells, ${ }^{1}$ and it also works as a scaffold for the cell growth. The use of PRGF-membrane can increase the anatomic and visual results in the surgical repair of recurrent MHs; no complications were observed after its use. PRGFmembrane can be used as adjunctive therapy for the treatment of recurrent large myopic MHs.

In conclusion, the placement of PRGF-membrane inside the hole can improve anatomic and visual results in the surgical repair of recurrent $\mathrm{MHs}$, helping to restore the anatomy of the macular area. The present clinical case could open the door to new investigations in the retina, aimed at the process of tissue regeneration.

\section{Ethical approval}

The principles of the Declaration of Helsinki were fulfilled. The informed consent for the use of the PRGF, and for the publication of the results obtained in the clinical case were signed by the patient.

\section{Abbreviation list}

EGF, epidermal growth factor; IGF, insulin-like growth factor; ILM, internal limiting membrane; $\mathrm{MH}$, macular hole; PDGF, platelet-derived growth factor; PRGF, plasma rich in growth factors; PPV, pars plana vitrectomy; VA, visual acuity; TGF- $\beta$, transforming growth factor- $\beta$.

\section{Acknowledgments}

Francisco Muruzabal and Virginia Cuadrado for their contribution to the revision of the manuscript. The development of this study did not have funding funds. Presented as clinical case in the congress of the European School for Advanced Studies in Ophthalmology (ESASO). Portugal 2016.

\section{Disclosure}

The authors report no conflicts of interest in this work.

\section{References}

1. Figueroa MS, Govetto A, Arriba-Palomero PD. Short-term results of platelet-rich plasma as adjuvant to $23-\mathrm{G}$ vitrectomy in the treatment of high myopic macular holes. Eur J Ophthalmol. 2015;1-6. doi:10.5301/ ejo.5000729

2. Wu -L-L, Ho T-C, Yang C-H, Yang C-M. Vitreo-retinal relationship and post-operative outcome of macular hole repair in eyes with high myopia. Graefes Arch Clin Exp Ophthalmol. 2016;254(1):7-14. doi:10.1007/s00417-015-2986-2

3. Wu -T-T, Kung Y-H. Comparison of anatomical and visual outcomes of macular hole surgery in patients with high myopia vs. non-high myopia: a case-control study using optical coherence tomography. Graefes Arch Clin Exp Ophthalmol. 2012;250(3):327-331. doi:10.1007/s00417-011-1821-7

4. Madi HA, Masri I, Steel DH. Optimal management of idiopathic macular holes. Clin Ophthalmol. 2016;10:97-116. doi:10.2147/OPTH.S96090

5. Anitua E, Muruzabal F, Pino A, Merayo-Lloves J, Orive G. Biological stability of plasma rich in growth factors eye drops after storage of 3 months. Cornea. 2013;32(10):1380-1386. doi:10.1097/ ICO.0b013e31829f7088

6. Anitua E, Muruzabal F, Tayebba A, et al. Autologous serum and plasma rich in growth factors in ophthalmology: preclinical and clinical studies. Acta Ophthalmol. 2015:1-10. doi:10.1111/ aos. 12710

7. Riestra AC, Vazquez N, Chacon M, et al. Autologous method for ex vivo expansion of human limbal epithelial progenitor cells based on plasma rich in growth factors technology. Ocul Surf. 2017;15(2):248256. doi:10.1016/j.jtos.2017.01.003

8. Sanchez-Avila RM, Merayo-Lloves J, Riestra AC, et al. Plasma rich in growth factors membrane as coadjuvant treatment in the surgery of ocular surface disorders. Medicine (Baltimore). 2018;97(17):e0242. doi:10.1097/MD.0000000000010242 
9. Merayo-Lloves J, Sanchez RM, Riestra AC, et al. Autologous plasma rich in growth factors eyedrops in refractory cases of ocular surface disorders. Ophthalmic Res. 2015;55(2):53-61. doi:10.1159/000439280

10. Coppola M, Rabiolo A, Cicinelli MV, Querques G, Bandello F. Vitrectomy in high myopia: a narrative review. Int J Retin Vitr. 2017;3(1):37. doi:10.1186/s40942-017-0090-y

11. Michalewska Z, Michalewski J, Dulczewska-Cichecka K, Nawrocki J. Inverted internal limiting membrane flap technique for surgical repair of myopic macular holes. Retina. 2014;34(4):664-669. doi:10.1097/IAE.0000000000000042

12. Alkabes M, Pichi F, Nucci P, et al. Anatomical and visual outcomes in high myopic macular hole (HM-MH) without retinal detachment: a review. Graefe's Arch Clin Exp Ophthalmol. 2014;252(2):191-199. doi:10.1007/s00417-013-2555-5
13. Anitua E, Prado R, Orive G. Closing regulatory gaps : new ground rules for platelet-rich plasma. Trends Biotechnol. 2015;33(9):492495. doi:10.1016/j.tibtech.2015.07.002

14. Sanchez-Avila RM, Merayo-Lloves J, Riestra AC, et al. The effect of immunologically safe plasma rich in growth factor eye drops in patients with sjogren syndrome. J Ocul Pharmacol Ther. 2017. doi:10.1089/jop.2016.0166

15. Anitua E, Muruzabal F, de la Fuente M, Merayo J, Duran J, Orive G. plasma rich in growth factors for the treatment of ocular surface diseases. Curr Eye Res. 2016;41(7):875-882. doi:10.3109/ 02713683.2015 .1104362

16. Anitua E, Prado R, Azkargorta M, et al. High-throughput proteomic characterization of plasma rich in growth factors (PRGF-Endoret)derived fibrin clot interactome. J Tissue Eng Regen Med. 2015;9(11): E1-12. doi:10.1002/term.1721

\section{Publish your work in this journal}

The International Medical Case Reports Journal is an international, peer-reviewed open-access journal publishing original case reports from all medical specialties. Previously unpublished medical posters are also accepted relating to any area of clinical or preclinical science. Submissions should not normally exceed 2,000 words or 4 published pages including figures, diagrams and references. The manuscript management system is completely online and includes a very quick and fair peer-review system, which is all easy to use. Visit http://www.dovepress.com/testimonials.php to read real quotes from published authors. 